This is the author's copy of the publication as archived in the DLR electronic library at http://elib.dlr.de. Please consult the original publication for citation, see https://arc.aiaa.org/doi/10.2514/6.2018-3150.

\title{
Aeroservoelastic Modeling and Analysis of a Highly Flexible Flutter Demonstrator
}

\author{
Matthias Wüstenhagen, Thiemo Kier, Manuel Pusch, Daniel Ossmann, Muhammad Yasser
} Meddaikar and Andreas Hermanutz

In this paper, the aeroservoelastic modeling of a highly flexible flutter demonstrator is presented. A finite element model of the demonstrator is generated and condensed to a reduced number of degrees of freedom to represent the structural dynamics. The unsteady aerodynamics are captured by the doublet lattice method based on potential theory. By interconnection of structural dynamics and unsteady aerodynamics an aeroelastic model is derived, which provides a basis for the design of a flutter suppression controller. In order to enable an efficient flutter suppression a clear separation of the occurring flutter mechanisms in speed and frequency is desired. To achieve his, the positions of the actuators controlling the flaps are varied within the scope of the aircraft design process. Due to their large mass contribution, the placement of the actuators has a crucial impact on the overall flutter characteristics and optimal actuator positions are determined by means of a mass sensitivity study.

\section{Copyright Notice}

Copyright (C) 2018 by German Aerospace Center (DLR). Published by the American Institute of Aeronautics and Astronautics, Inc., with permission.

Wüstenhagen, M., Kier, T., Meddaikar, Y. M., Pusch, M., Ossmann, D., and Hermanutz, A., "Aeroservoelastic Modeling and Analysis of a Highly Flexible Flutter Demonstrator," 2018 Atmospheric Flight Mechanics Conference, American Institute of Aeronautics and Astronautics, Reston, Virginia, 2018. https://doi.org/10.2514/6.2018-3150 


\title{
Aeroservoelastic Modeling and Analysis of a Highly Flexible Flutter Demonstrator
}

\author{
Matthias Wüstenhagen*, Thiemo Kier, Manuel Pusch and Daniel Ossmann \\ Institute of System Dynamics \& Control \\ German Aerospace Center (DLR), 82234 Weßling, Germany \\ Muhammad Yasser Meddaikar \\ Institute of Aeroelasticity \\ German Aerospace Center (DLR), з7073 Göttingen, Germany \\ Andreas Hermanutz \\ Institute of Aircraft Design \\ Technical University of Munich (TUM), 85748 Garching bei München, Germany
}

\begin{abstract}
In this paper, the aeroservoelastic modeling of a highly flexible flutter demonstrator is presented. A finite element model of the demonstrator is generated and condensed to a reduced number of degrees of freedom to represent the structural dynamics. The unsteady aerodynamics are captured by the doublet lattice method based on potential theory. By interconnection of structural dynamics and unsteady aerodynamics an aeroelastic model is derived, which provides a basis for the design of a flutter suppression controller. In order to enable an efficient flutter suppression a clear separation of the occurring flutter mechanisms in speed and frequency is desired. To achieve this, the positions of the actuators controlling the flaps are varied within the scope of the aircraft design process. Due to their large mass contribution, the placement of the actuators has a crucial impact on the overall flutter characteristics and optimal actuator positions are determined by means of a mass sensitivity study.
\end{abstract}

\section{Introduction}

The fuel costs account for the greatest portion of the operating costs of today's aircraft. Therefore, fuel efficiency is one of the most important aspect of new aircraft design concepts. ${ }^{1}$ Modifications to the wings hold great potential to increase fuel efficiency. Lightweight wing structures and higher wing aspect ratios for better aerodynamic efficiency are changes that can make flying more economic. These modifications, however, lead to a higher flexibility of the wing structure and therefore reduce the flutter speed. Flutter is a vibrational instability of the wings, which can result in catastrophic structural failure. It generally involves the coupling between wing bending and torsion. ${ }^{2,3}$ To prevent flutter and guarantee stability within the entire flight envelope, active flutter suppression can be used. Within the Horizon 2020 project Flutter Free FLight Envelope eXpansion for ecOnomic Performance improvement (FLEXOP) active flutter suppression methods are developed and are tested on the demonstrator aircraft shown in Figure 1. The heart of the active flutter suppression system is a flutter controller, which controls actuators connected to the ailerons. The development of an active flutter control system is based on aeroelastic models. It is therefore essential to derive sophisticated aeroelastic models beforehand. More insight into the design of an active flutter controller is given in Ref. 5 .

The modeling process within the FLEXOP project is described in Figure 2. A finite element (FE) model is used for representing the structural dynamics of the demonstrator. However, a FE model typically

${ }^{*}$ Corresponding Author, Research Fellow, matthias.wuestenhagen@dlr.de 


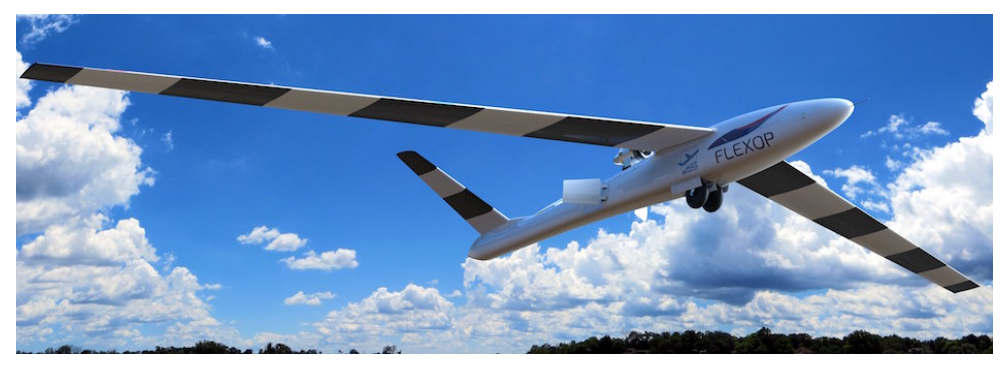

Figure 1: FLEXOP demonstrator aircraft ${ }^{4}$

features many degrees of freedom (DOF) which makes it unsuitable for flutter control design. By means of the Guyan reduction the DOF are reduced while the relevant dynamics of the system are maintained. This results in a condensed model with significantly less DOF. Based on this model the equations of motion $(\mathrm{EOM})$ describing the rigid and flexible aircraft motion are formulated. In Section II the EOM representing the structural dynamics are introduced.

The aerodynamic model is based on a set of trapezoidal aerodynamic boxes, which represent the lifting surfaces. By applying either vortices or doublets to all boxes, the flow field of the demonstrator is approximated using the potential theory. With the application of vortices this approach is called vortex lattice method (VLM), while with doublets it is known as the doublet lattice method (DLM), respectively. While the VLM represents steady aerodynamics, the DLM also takes unsteady aerodynamic effects into account. For the DLM, extra states known as lag states are introduced. Modeling unsteady aerodynamics enlarges the model size significantly, which makes it more difficult to derive suitable aerservoelastic models for controller synthesis. Therefore, in some cases it might be more suitable to focus on steady aerodynamics and use the VLM instead, especially when unsteady effects are negligible. However, for active flutter control unsteady aerodynamic effects have to be considered by using the DLM. ${ }^{2,6}$ The physics behind the VLM and the DLM are described in Section III.

The interconnection between the structural dynamics and aerodynamics is carried out through splining, which results in an aeroelastic model. This process is described in more detail in Section IV. Within the scope of this paper, the aeroservoelastic tool chain is modified to support the aircraft design process with respect to the positioning of the actuators for flutter suppression. In Ref. 7 the aircraft design process of the demonstrator aircraft is described in more detail. Due to inertial forces, the position of the actuators greatly affects the overall flutter behavior. A mass sensitivity study is performed to analyze the effect of different positions on the overall open-loop system performance. A set of aeroelastic models featuring different actuator positions is evaluated with respect to their flutter behavior. Two different flutter mechanisms symmetric and asymmetric - can be observed. For an efficient active flutter suppression the separation in flutter speed and frequency of these two flutter mechanisms is maximised by the actuator placement as shown in Section V. The observability and controllabiltiy of the occurring flutter modes are assumed as given. Further investigations on the observability and controllability of the flutter modes are presented in Ref. 8. This paper thus presents a comprehensive approach on how the actuator placement can help optimize the open-loop flutter behavior.

\section{Structural Dynamics}

The structural dynamics of the flexible aircraft can be divided into a rigid-body and an elastic-body motion. The rigid-body motion basically describes the maneuver characteristics of the aircraft. In contrast, the elastic-body motion represents the dynamics due to the flexibility of the aircraft structure. While the rigid-body motion is described in nonlinear form, the equation of the elastic-body motion is considered to be linear. A detailed FE model serves as basis for the structural model of the aircraft. The process of generating the FE model and its condensed version is described below. Subsequently the EOM representing the rigid and elastic-body motion are defined for the condensed model. 


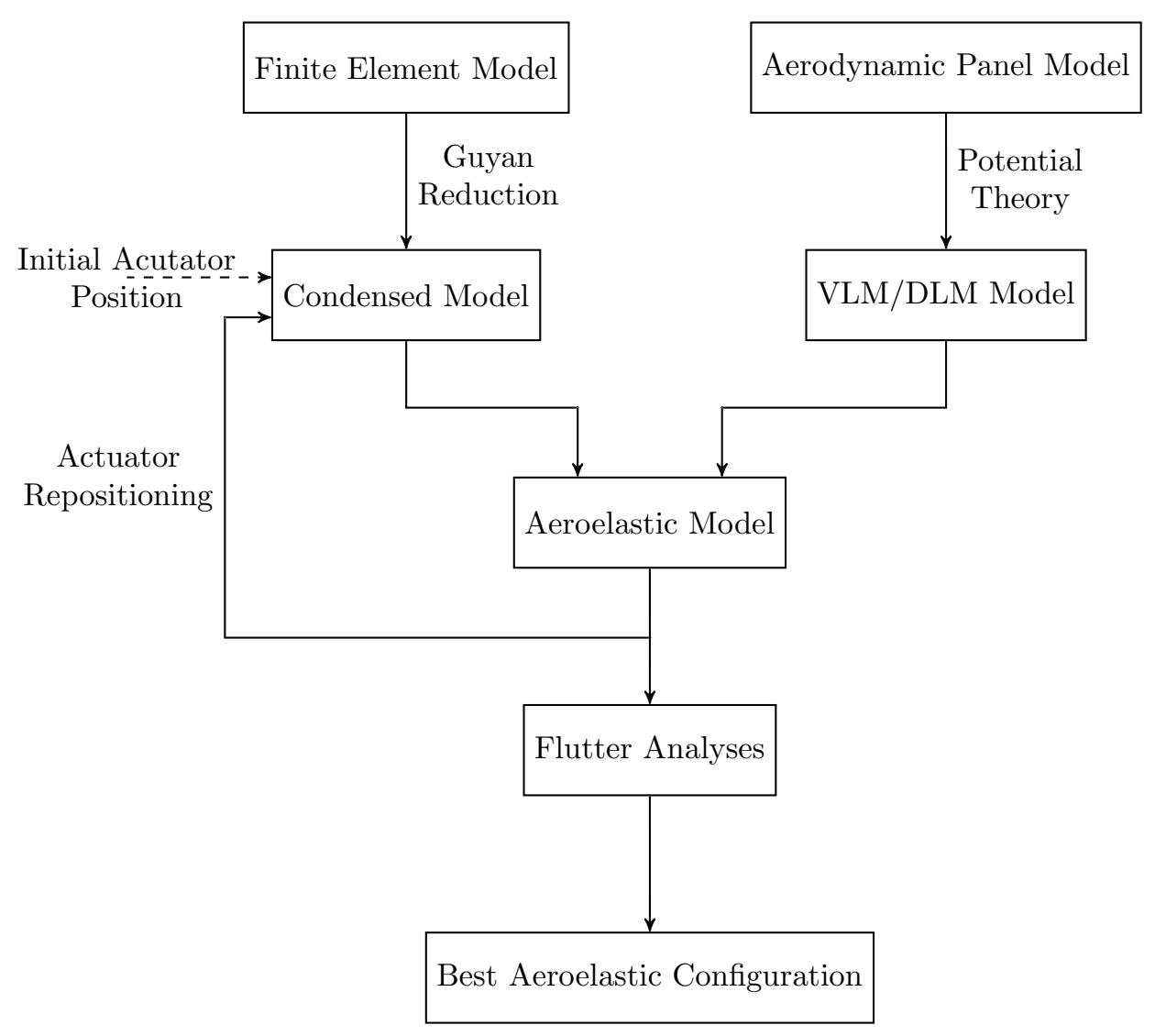

Figure 2: Modeling process of the demonstrator aircraft

\section{A. Finite Element Model}

The aircraft structural FE model comprises the wing, fuselage and empennage and is shown in Figure 3. The FE software used here is MSC.NASTRAN. The wing is represented by a high-fidelity FE model comprising beam, surface and solid elements. Rigid-body interpolation elements are added at pre-defined locations throughout the wing to facilitate the required model reduction. Furthermore, the wing mass model is density-based as opposed to a lumped mass model. The fuselage structure is modeled using beam elements. The equivalent beam stiffnesses are obtained utilizing the cross-sections of the fuselage hull at different sections and the lay-up of the hull. ${ }^{9}$ The mass is then lumped at the two beam nodes. The V-tail empennage FE model is shell-element based comprising of the main structural load-bearing entities - the upper and lower skins, structural rubs, spars and the non-structural masses. Similar to the wing FE model, a density-based mass representation is used for the empennage as well.

Given that the FE model of the wing is of very high-fidelity (more than 600000 nodes), a Guyan-reduction, also called condensation, is performed reducing the mass and stiffness matrix to 165 nodes in the condensed model. ${ }^{10}$ The condensation nodes are distributed as follows: 33 at the fuselage beam grid points, 12 in each of the V-tail halves, 72 in the main wing-box facilitated by the rigid body interpolation elements mentioned earlier and 7 points per flap, of which each wing possesses 4 .

For the mass sensitivity study, the actuators for active flutter suppression need to be added to the condensed model as their inertial properties have a significant impact on the system performance. The actuators are assumed to be cylindrical featuring a homogenous mass distribution as shown in Figure 4. Their mass matrix is defined by

$$
M_{a}=\operatorname{diag}\left(m_{a}, m_{a}, m_{a}, \frac{1}{4} m_{a} r_{a}^{2}+\frac{1}{12} m_{a} l_{a}^{2}, \frac{1}{2} m_{a} r_{a}^{2}, \frac{1}{4} m_{a} r_{a}^{2}+\frac{1}{12} m_{a} l_{a}^{2}\right),
$$

where $r_{a}$ is the radius, $l_{a}$ the height and $m_{a}$ the mass of the actuators. ${ }^{11}$ The attachment of the actuators to 


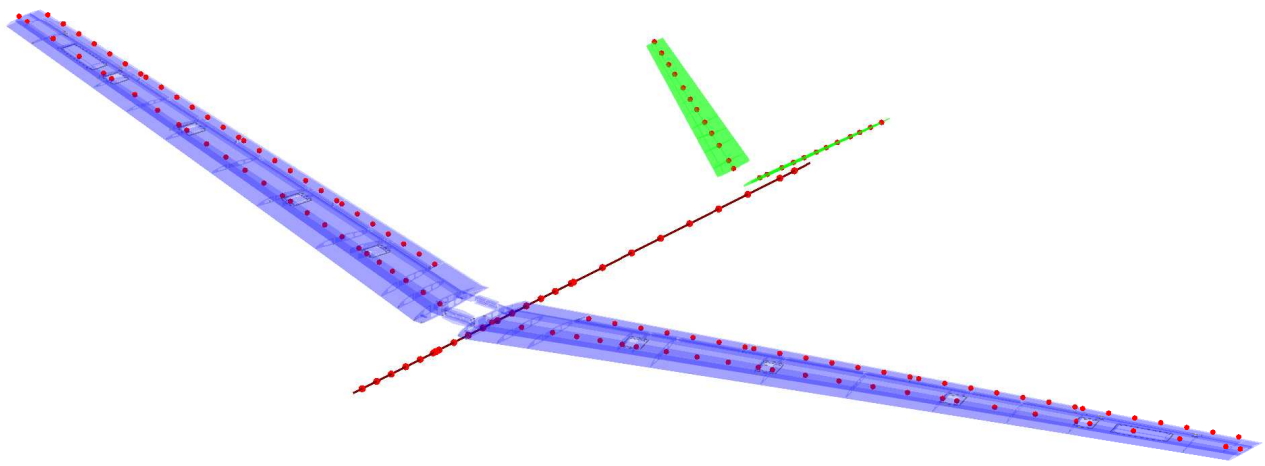

Figure 3: Full FE model of the FLEXOP demonstrator aircraft

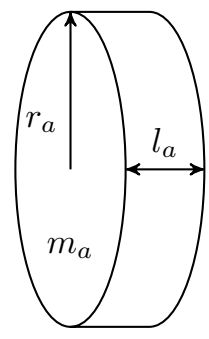

Figure 4: Actuator model

the condensed model is considered to be rigid, meaning that each actuator is stiffly connected to the closest condensation point. The actuator inertial loads are directly introduced into the condensed model.

\section{B. Equations of Motion}

The condensed model features rigid body and flexible modes, which are described by the EOM. The EOM are based on an equilibrium of forces and moments. They describe the behavior of the aircraft due to external loads originating from the aerodynamics and thrust. For simplification, the following assumptions are made.

1. As the earth rotation can be neglected, the inertial reference system is earth fixed. ${ }^{6}$

2. Gravity is constant over the airframe. ${ }^{12}$

3. The deformations of the airframe are considered to be small which allows the use of linear elastic theory defined by Hooke's law. ${ }^{6}$

4. Due to small deformations of the aircraft structure, the aircraft mass moment of inertia $J_{b}$ remains unchanged. ${ }^{6}$

5. As the structural deformations are small, loads act on the undeformed airframe. ${ }^{12}$

6. The eigenvectors of the modal analysis are orthogonal, because of which the total structural deformation can be written as a linear combination of the modal deflections. ${ }^{12}$

7. The rigid body and flexible body EOM are considered to be decoupled. ${ }^{12}$

\section{Rigid Body Motion}

For the derivation of the nonlinear flight mechanical EOM, the aircraft is considered as a rigid body with a constant mass $m_{b}$ and constant mass moment of inertia $J_{b}$. Therefore, the aircraft rigid-body motion is described by

$$
\begin{gathered}
{\left[\begin{array}{c}
m_{b}\left(\dot{V}_{b}+\Omega_{b} \times V_{b}-T_{b e} g_{e}\right) \\
J_{b} \dot{\Omega}_{b}+\Omega_{b} \times\left(J_{b} \Omega_{b}\right)
\end{array}\right]=\Phi_{g b}^{T} P_{g}^{\mathrm{ext}}(t),} \\
\frac{4 \text { of } 12}{\text { American Institute of Aeronautics and Astronautics }}
\end{gathered}
$$


which represents the Newton-Euler EOM. ${ }^{13}$ In Equation 2 the translational and angular velocity of the aircraft with respect to the body frame of reference are given by $V_{b}$ and $\Omega_{b}$. The vector $g_{e}$ represents the gravitational acceleration, which is transformed with $T_{b e}$ from the earth fixed to the body fixed frame of reference. The external loads $P_{g}^{\text {ext }}(t)$ acting on the aircraft structure are transformed by the transpose of $\Phi_{g b}^{T}$ into the rigid body frame. ${ }^{6}$

\section{Elastic Body Motion}

As the displacements due to the aircraft flexibility are assumed to be small, linear elastic theory is applied to define the elastic motion. Therefore the correlation between external loads $P_{g}^{\text {ext }}(t)$ and the generalized coordinates $u_{f}$ representing the modal deformation of the structure is given by the differential equation

$$
M_{f f} \ddot{u}_{f}+B_{f f} \dot{u}_{f}+K_{f f} u_{f}=\Phi_{g f}^{T} P_{g}^{\mathrm{ext}}(t),
$$

where $M_{f f}, B_{f f}$ and $K_{f f}$ are the modal mass, damping and stiffness matrices. The modal matrix $\Phi_{g f}$ contains the eigenvectors of the structural modes sorted by frequency. ${ }^{6}$ Typically, higher frequencies have a smaller contribution to the overall system performance. Consequently, modal truncation can be applied to reduce the DOF significantly by considering only the most relevant eigenmodes.

\section{Aerodynamics}

The aerodynamic loads represent the major external loads acting on the aircraft structure. Their calculation is based on the VLM for steady aerodynamics and the DLM for unsteady aerodynamics. Both methods are based on a panel model, which is described in the following section.

\section{A. Panel Model}

The lifting surfaces are discretized by several trapezoidal shaped panels, known as aerodynamic boxes as shown in Figure 5. Of note is the panel model for the fuselage. The wetted areas of the fuselage are projected onto a T-cruciform shaped panel model. Although this is a vast simplification, the fuselage aerodynamics are modeled quite accurately with respect to higher-order CFD simulations.

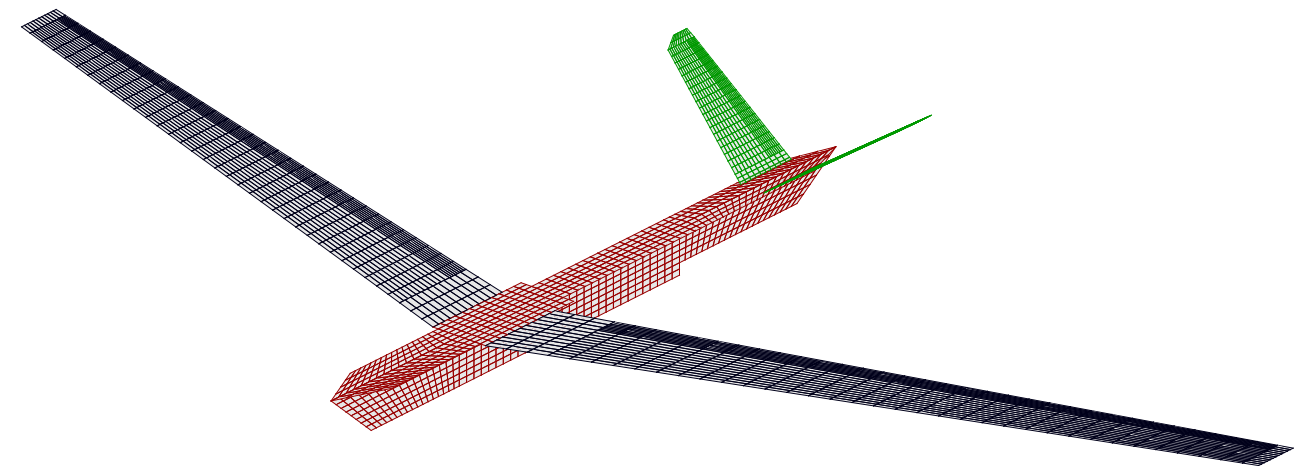

Figure 5: Aerodynamic boxes of the FLEXOP demonstrator aircraft

\section{B. Steady Aerodynamics via VLM}

The VLM is used to model steady aerodynamics. As can be seen in Figure 6a, each aerodynamic box of the panel model possesses a horseshoe vortex at point $l$ on the quarter-chord line. Due to the Helmholtz theorem the vortex is shed downstream to infinity at the side edges of the box. For each aerodynamic box the Pistolesi Theorem needs to be met, stating that there is no perpendicular flow through the control point $j$ at the three-quarter-chord line. Therefore the induced velocity at the control point needs to equalize the perpendicular component of the incoming flow, like shown in Figure 6b. By means of the Biot-Savart law 


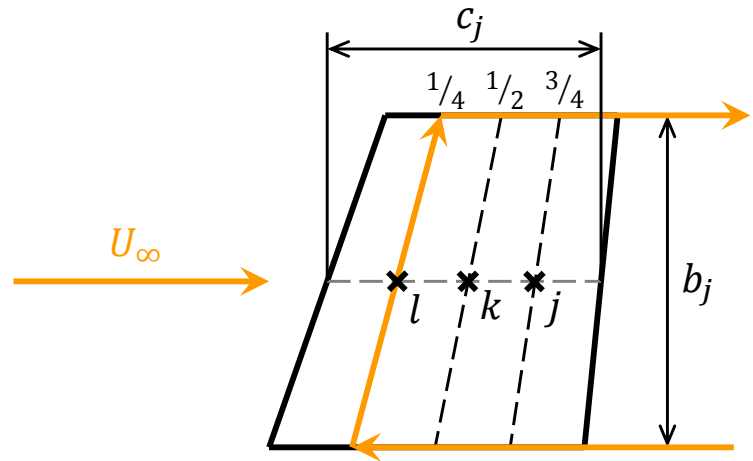

(a) Top view

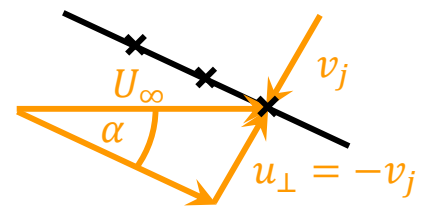

(b) Side view

Figure 6: Schematic drawing of an aerodynamic box

the induced velocities $v_{j}$ due to the circulation strengths $\Gamma_{j}$ of the horseshoe vortices can be determined by

$$
v_{j}=A_{j j} \Gamma_{j} .
$$

The matrix $A_{j j}$ describes the contribution of all vortices to the induced velocities of the aerodynamic boxes. Inverting $A_{j j}$ and multiplying with $2 / c_{j}$, where $c_{j}$ is the chord length of the respective aerodynamic box, leads to the aerodynamic influence coefficient (AIC) matrix $Q_{j j}$. It is used to determine the pressure coefficient

$$
\Delta c_{p j}=Q_{j j} w_{j},
$$

where $w_{j}=\frac{v_{j}}{U_{\infty}}$ is the downwash $v_{j}$ normalized with the flight speed $U_{\infty}$. It is assumed to be equal to the angle of attack $\alpha$, i.e. $w_{j}=\sin (\alpha) \approx \alpha$, as only small angles are considered.

There are different contributions, that determine the value of $w_{j}$. The VLM is based on lift coefficient gradients, meaning the change in lift can be predicted very well. In order to receive the absolute lift coefficients, they have to be initialized by a reference calculation. Therefore the downwash has to be corrected by means of $w_{j b_{0}}$. Within the FLEXOP project a steady computational fluid dynamics (CFD) calculation at an angle of attack $\alpha=0$ is used. Rigid-body motions of the aircraft also affect the downwash. For example, a downward motion of the aircraft changes the direction of the incoming flow and therefore the angle of attack. $w_{j b_{1}}$ accounts for the contribution due to a rigid-body motion. Also the deflection of the control surfaces affects the steady aerodynamics. By deflecting the aerodynamic boxes, belonging to the control surfaces, the downwash is changed by $w_{j x_{0}}$. Not only the deflection of the control surfaces alters the lift, but also their deflection rate, which can be accounted for by $w_{j x_{1}}$.

So far only aerodynamic effects of a rigid-body have been considered. In order to consider aeroelastic effects, the downwash has to be dependent on flexible motions. Comparable to the control surfaces, the flexible deflection and deflection rate of the aircraft contribute with $w_{j f_{0}}$ and $w_{j f_{1}}$ to the downwash. ${ }^{6,14-16}$ Finally the equation for the downwash is given by

$$
w_{j}=w_{j b_{0}}+w_{j b_{1}}+w_{j x_{0}}+w_{j x_{1}}+w_{j f_{0}}+w_{j f_{1}} .
$$

\section{Unsteady Aerodynamics via DLM}

In order to take unsteady aerodynamic effects into account, the aerodynamic model has to be widened. When an airfoil is suddenly moved forward at an angle of attack, it creates circulation. Since the Helmholtz theorem states that the total circulation has to stay constant, a vortex of the same strength but with opposite direction of rotation has to be shed from the trailing edge. The vortex moves downstream losing its influence on the airfoil the farther away it gets. Its effect therefore decreases with time and the flow converges to the steady condition. This lagging effect is caused by unsteady aerodynamics. ${ }^{14}$

Unsteady aerodynamics are covered by the Doublet Lattice Method (DLM). Instead of horseshoe vortices, doublets are placed at the quarter-chord line of each aerodynamic box. In contrast to the VLM, the pressure coefficient is determined by

$$
\Delta c_{p j}(k)=Q_{j j}(k) w_{j}(k)
$$


in the reduced frequency domain, where the dimensionless reduced frequency is

$$
k=\omega \frac{c_{r} / 2}{U_{\infty}} .
$$

In Equation 8, $c_{r}$ depicts the reference chord length and $\omega$ is the frequency. For $k=0$ the quasi-steady solution is derived. In order to be able to transform the unsteady aerodynamics to the time domain, a rational function approximation (RFA) is chosen. The AIC matrix is approximated with Roger's method, which is described in detail in Ref. 17, as

$$
Q_{j j}\left(s^{\star}\right)=Q_{0, j j}+Q_{1, j j} s^{\star}+\sum_{i=1}^{n_{p}} Q_{L_{i}, j j} \frac{s^{\star} I}{s^{\star}+p_{i}},
$$

where $s^{\star}=i k$ is equivalent to the Laplace variable $s$ for the reduced frequency $k$. When multiplying with the downwash $w_{j}$ from the right side and performing an inverse Laplace transformation, Equation (9) becomes

$$
\begin{aligned}
& \Delta c_{p j}=\mathcal{L}^{-1}\left(Q_{j j}\left(s^{\star}\right) w_{j}\left(s^{\star}\right)\right) \\
& =\underbrace{Q_{0, j j} w_{j}}_{\text {quasi-steady }}+\underbrace{\left(Q_{1, j j}+\left[\begin{array}{lll}
Q_{L_{1}, j j} & \ldots & Q_{L_{n_{p}}, j j}
\end{array}\right] \mathcal{L}^{-1}\left(\sum_{i=1}^{n_{p}} \frac{Q_{L_{i}, j j}}{s^{\star}+p_{i}}\right)\right)\left(\frac{c_{r} / 2}{U_{\infty}}\right) \dot{w}_{j}}_{\text {unsteady }} .
\end{aligned}
$$

The unsteady aerodynamics can be represented as a state space system, defined by

$$
\begin{aligned}
\dot{x}_{L} & =A x_{L}+B \dot{w}_{j} \\
\Delta c_{p j_{\text {unsteady }}} & =C x_{L}+D \dot{w}_{j} \\
A & =\operatorname{diag}\left(\left[\begin{array}{lll}
-p_{1} I & \ldots & -p_{n_{p}} I
\end{array}\right]\right)\left(\frac{U_{\infty}}{c_{r} / 2}\right) \\
B & =\left[\begin{array}{lll}
I & \ldots & I
\end{array}\right]^{T} \\
C & =\left[\begin{array}{lll}
Q_{L_{1}, j j} & \ldots & Q_{L_{n_{p}}, j j}
\end{array}\right] \\
D & =Q_{1, j j}\left(\frac{c_{r} / 2}{U_{\infty}}\right) .
\end{aligned}
$$

The new states $x_{L}$ are called lag states. They represent the lagging behavior, which is caused by unsteady aerodynamics.

It can be seen, that the first term in Equation (10) depends on the downwash $w_{j}$, while the second term depends on its derivative $\dot{w}_{j}$. Therefore, it can be clearly distinguished between quasi-steady and unsteady aerodynamics. By differentiating Equation (6) $\dot{w}_{j}$ is obtained by

$$
\dot{w}_{j}=\dot{w}_{j b_{0}}+\dot{w}_{j b_{1}}+\dot{w}_{j x_{0}}+\dot{w}_{j x_{1}}+\dot{w}_{j f_{0}}+\dot{w}_{j f_{1}},
$$

where $w_{j b_{0}}$ is considered to be constant and therefore its derivative $\dot{w}_{j b_{0}}$ is equal to zero. The rigid-body accelerations, the control surface deflection rates and accelerations as well as the flexible-body deflection rates and accelerations cause unsteady aerodynamic effects and therefore a change in the downwash. ${ }^{6}$

\section{Model Integration}

The overall aeroservoelastic model is built of several sub-models, as depicted in Figure 7. The core forms the aeroelastic system, which represents the coupling of aerodynamics and structural dynamics. The aerodynamic loads $P_{g}^{\text {aero }}$ directly correlate with the structural motion and can be expressed in terms of $\Delta c_{p j}$ as

$$
P_{g}^{\text {aero }}=q_{\infty} T_{k g}^{T} S_{k j} \Delta c_{p j},
$$

where $S_{k j}$ is an integration matrix relating the pressure in the aerodynamic boxes at point $j$ with the forces at the aerodynamic grid points $k$. The forces at the aerodynamic grid points $k$ are then interpolated onto 


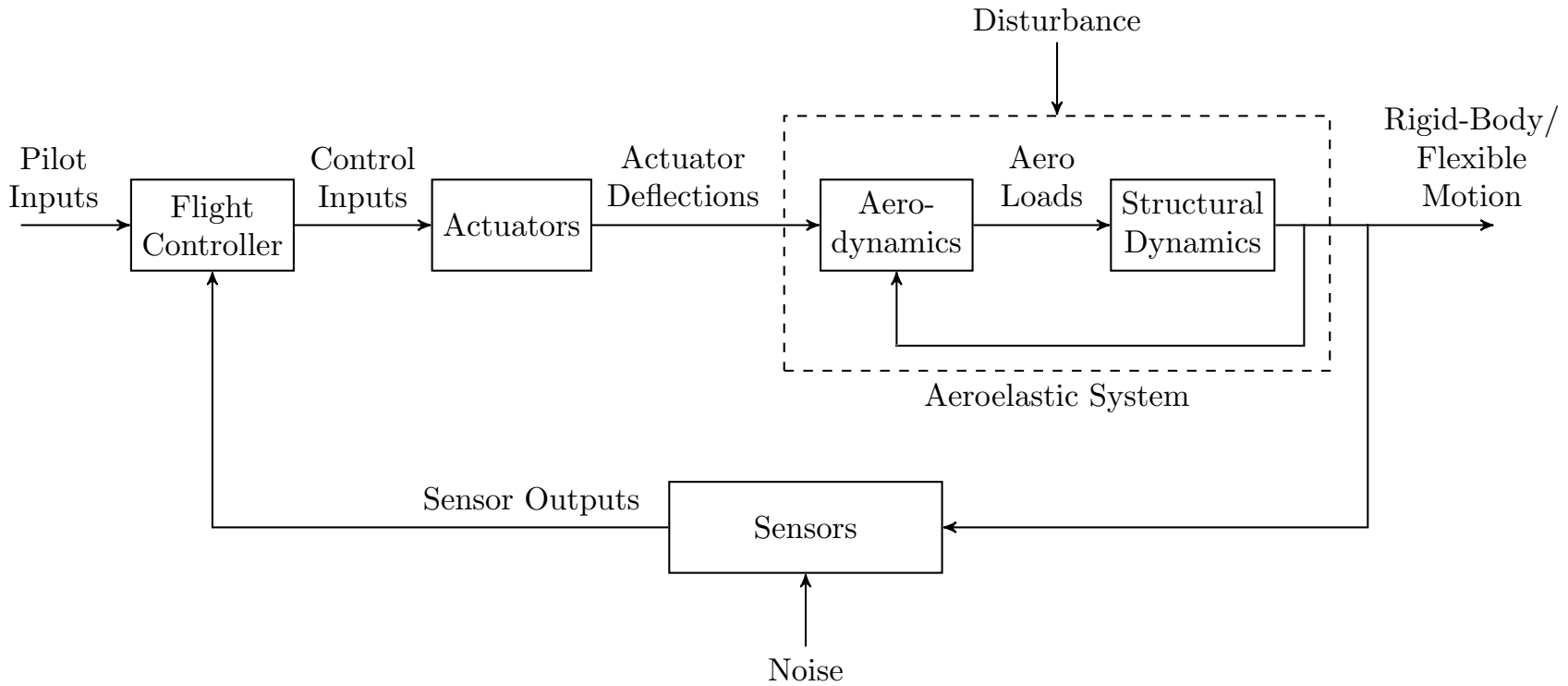

Figure 7: Aeroservoelastic system ${ }^{18}$

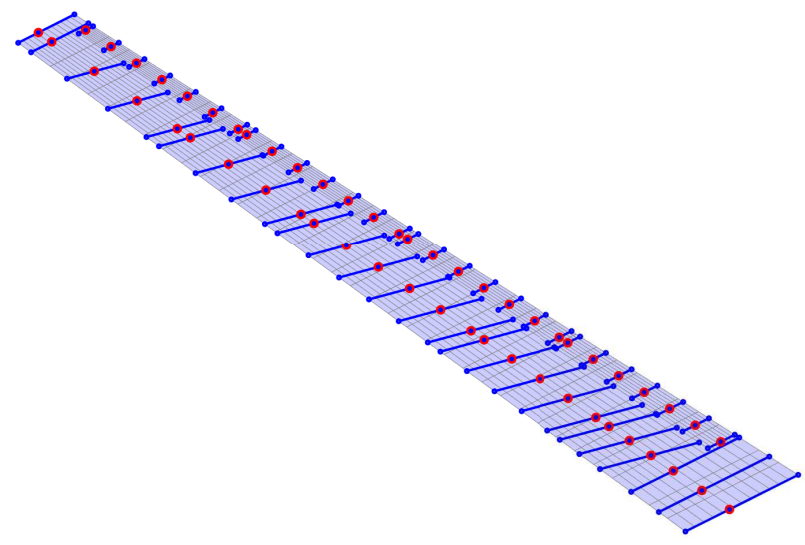

Figure 8: Splining between the aerodynamic model and structural model of the right wing

the structural grid points via the transpose of the spline matrix $T_{k g}$. The splining model for the wing is shown as an example in Figure 8. The structural nodes used for the splining comprise of the condensation nodes as well as two nodes per condensation node, extended to the leading and trailing edge at that point using rigid elements. A similar splining model is used for the empennage as well. Multiplying with the dynamic pressure $q_{\infty}$ leads then to the aerodynamic loads acting on the structure. The aircraft structure reacts on the aerodynamic loads by performing rigid-body and flexible motions, which directly affect the aircraft aerodynamics. Therefore the aeroelastic model is considered as a loop between strcutural dynamics and aerodynamics. ${ }^{6,18}$ An aeroservoelastic model is derived by adding an active flutter control system to the aeroelastic model. The active flutter control system comprises of actuators for deflecting the relevant control surfaces, sensors for observing the dynamic behavior of the aircraft and the flutter controller. As the actuators introduce additional inertial loads to the aircraft structure, they need to be considered during the aeroelastic design process of the aircraft. In the following section the flutter characteristics of the aeroelastic model are evaluatated with respect to the actuator position. 


\section{Model Analysis}

The main goal of the FLEXOP project is to increase the flutter speed by means of active flutter suppression techniques. To demonstrate active flutter suppression, the open-loop aeroelastic system needs to be designed in a way that the occuring flutter can be controlled. Thus, the aircraft design process is tailored to achieve a separation of the flutter phenomena in terms of frequencies and speeds. The poles of the open-loop aircraft, without considering the actuator masses, are visualized for a speed range of $45 \mathrm{~m} / \mathrm{s}$ to $70 \mathrm{~m} / \mathrm{s}$ in Figure 9. With increasing speed, two aeroelastic modes representing symmetric and asymmetric

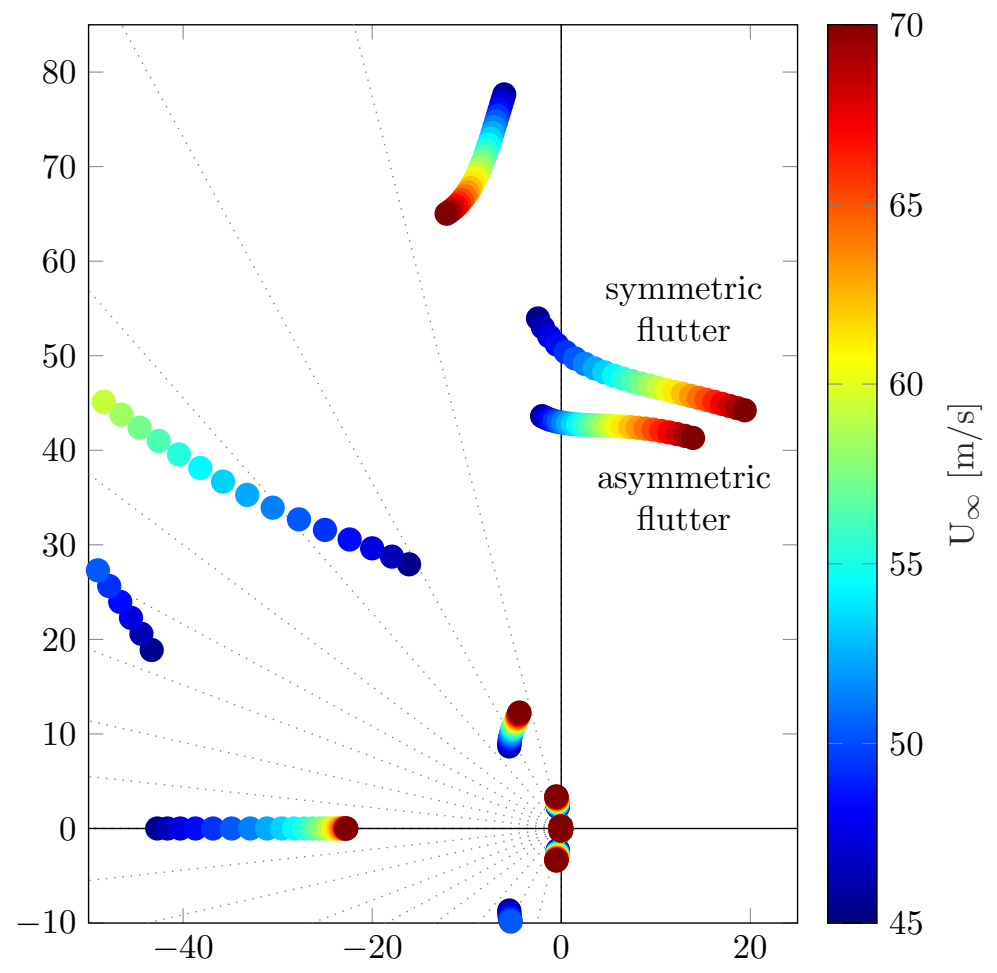

Figure 9: Aeroelastic poles

flutter, cross the imaginary axis and become unstable. The flutter speed of symmetric flutter is determined to be $48.51 \mathrm{~m} / \mathrm{s}$ at a frequency of $8.08 \mathrm{~Hz}$. In comparison asymmetric flutter becomes unstable at $50.58 \mathrm{~m} / \mathrm{s}$ and $6.83 \mathrm{~Hz}$. This demonstrates that the flutter characteristics are very close to each other in frequency and speed. Therefore, the idea is to separate the flutter phenomena for an easier flutter controller design by using the position of the actuators for flutter control as design freedom. The separation of the flutter frequencies allows designing decoupled flutter controllers for each flutter phenomenon. The separation in the flutter speeds helps to examine the stabilization of one flutter mode without having an interaction of the second flutter mode.

As the inertial forces introduced by the flutter actuators, significantly affect the aeroelastic poles, a mass sensitivity study is performed in order to analyze the change in the flutter characteristics due to different actuator positions. Figure 10 depicts the 15 examined actuator positions for the right wing. Linear interpolation of the resulting flutter speeds and frequencies between these 15 discrete points provides more insight in the dependence of flutter frequencies and speeds on the actuator position. Figure 11a depicts the symmetric flutter speed for different actuator positions. The flutter speed increases when placing the actuator close to the wing tip leading edge. However, the sensitivity with respect to the actuator placement is high at the wing tip, as small variations of the actuator position have a significant effect. The closer the actuators are placed towards the wing root, the less the symmetric flutter speed is affected. In Figure 12a the dependency of the asymmetric flutter speed on the actuator position is shown. The asymmetric flutter speed tends to increase as the actuator is moved towards the wing tip leading edge as well. However, it is mainly affected by chordwise changes of the actuator position. The frequency of symmetric and asymmetric flutter, depicted in Figure 11b and Figure 12b, increases for actuators placed towards the wing root. A 


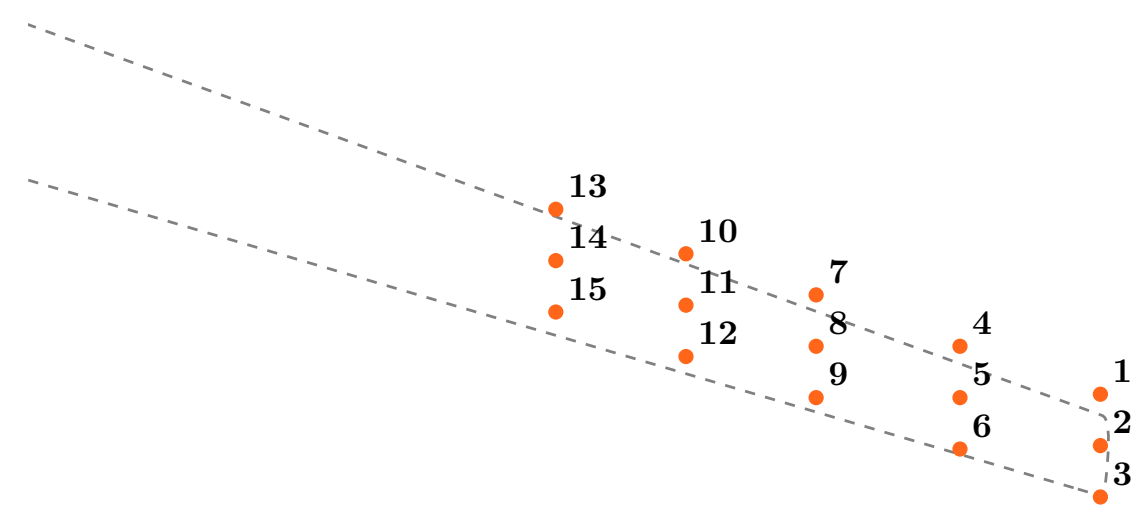

Figure 10: Actuator positions for mass sensitivity study (right wing, top view)

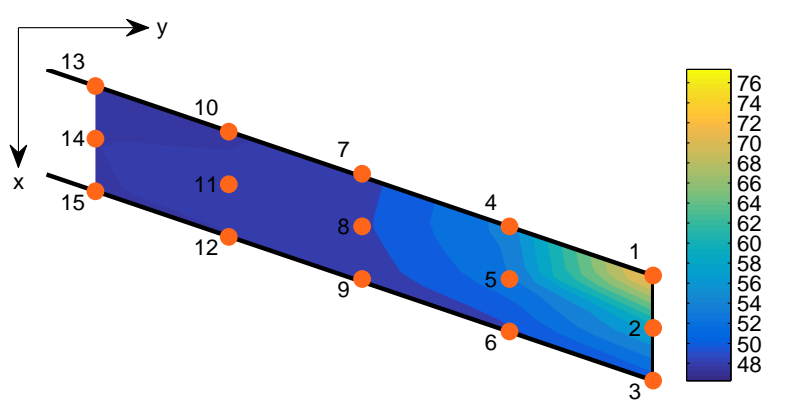

(a) Flutter speed in $m / s$

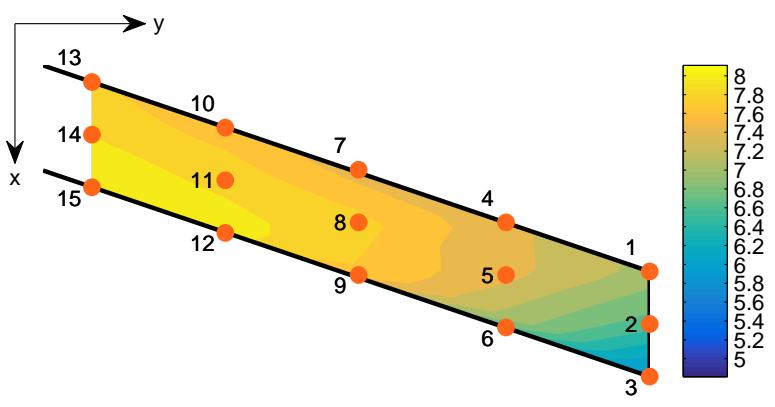

(b) Flutter frequency in $\mathrm{Hz}$

Figure 11: Symmetric flutter characteristics for different actuator positions (right wing, top view)

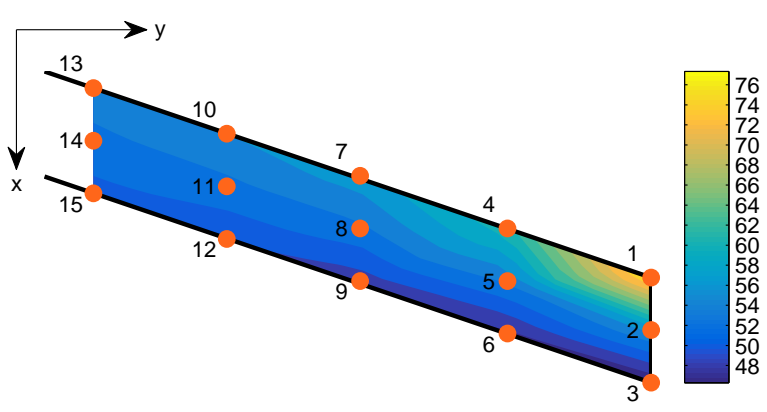

(a) Flutter speed in $m / s$

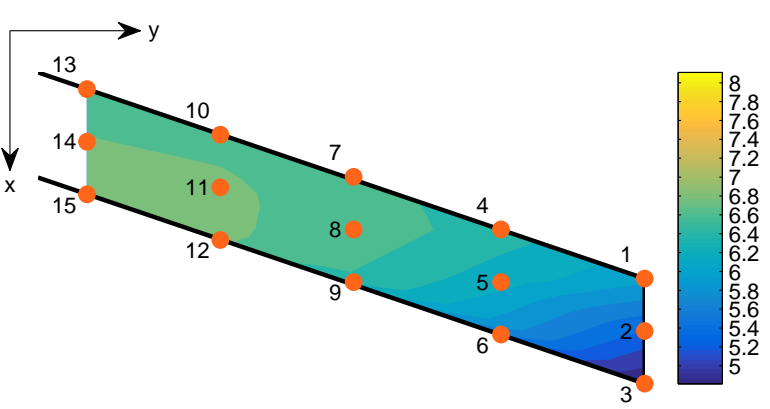

(b) Flutter frequency in $\mathrm{Hz}$

Figure 12: Asymmetric flutter characteristics for different actuator positions (right wing, top view)

minimum frequency is reached at the trailing edge of the wing tip.

The visualization of the absolute differences of the symmetric and asymmetric flutter characteristics with respect to the actuator position is shown in Figure 13. It allows to evaluate the separation in flutter speed and frequency. The separation in flutter speeds mainly depends on the actuator position in $x$-direction. Around actuator position 2,5 and 9 the flutter speeds match. The best possible separation in flutter speeds is achieved at actuator position 13. The difference in flutter frequencies is affected by the chordwise position of the actuators. It slightly increases from the leading to the trailing edge. However, the achievable separation in the flutter frequencies cannot be significantly changed by varying the actuator position. Thus, the decision on where to place the actuators is solely based on the examination of the separation in flutter speeds. It is 
therefore decided to attach the actuators at position 13 featuring the highest separation in flutter speeds.

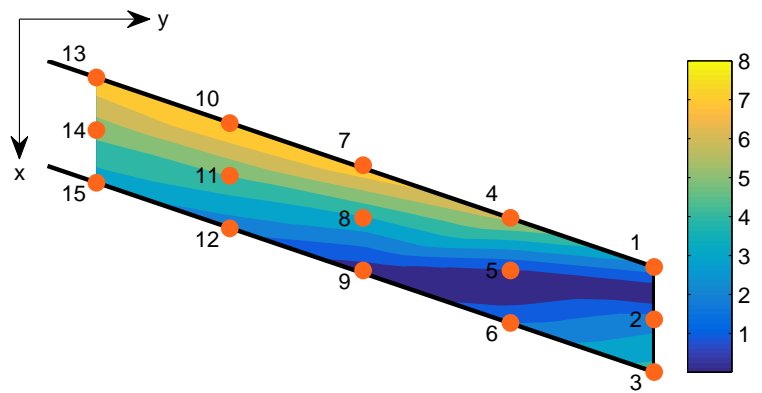

(a) Flutter speed separation in $\mathrm{m} / \mathrm{s}$

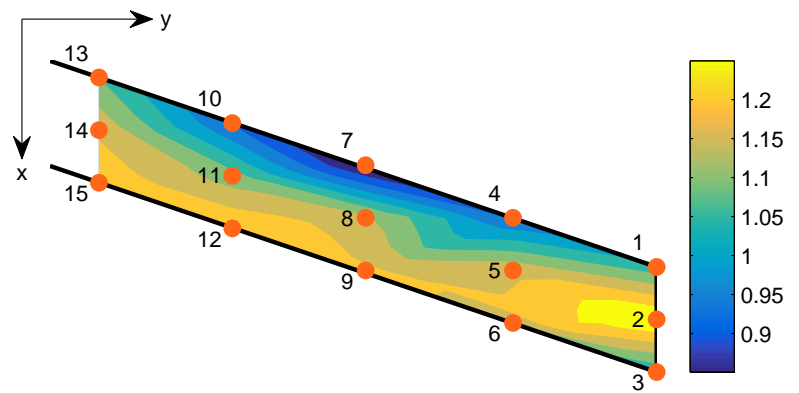

(b) Flutter frequency separation in $\mathrm{Hz}$

Figure 13: Separation in flutter characteristics for different actuator positions (right wing, top view)

\section{Conclusion}

In this paper, the aeroelastic modeling process for the application of active flutter techniques in a flying demonstrator is explained. While the rigid-body motion is described by the non-linear Newton-Euler equations, the flexible motion is based on linear elastic theory. The VLM is used to model the steady aerodynamics of the demonstrator. Since in some cases unsteady aerodynamic effects are significant, the aerodynamic model is widened by means of the DLM. An interconnection between the structural dynamics and aerodynamics is established via splining. The positions of the actuators for active flutter suppression are used as a design freedom to shape the open-loop flutter behavior. It is found that mainly the flutter speeds are affected by the actuator positions, while the differences in flutter frequencies remain nearly unchanged. By determining the actuator position with the methods presented in this paper the aeroelastic model is ready to be used for active flutter control design. This control design completes the aeroservoelastic tool chain for the FLEXOP demonstrator and will be carried out in future work.

\section{Acknowledgment}

The research leading to these results is part of the FLEXOP project. This project has received funding from the European Unions Horizon 2020 research and innovation program under grant agreement No. 636307. 


\section{References}

${ }^{1}$ International Energy Agency, Transport Energy and CO2: Moving Towards Sustainability, OECD Publishing, 2009.

${ }^{2}$ Dowell, E. H., A modern course in aeroelasticity, Vol. 217, Springer, 2014.

${ }^{3}$ Bisplinghoff, R., Ashley, H., and Halfman, R., "Aeroelasticity," Addison-Wes-ley Pub. Co, 1955.

${ }^{4}$ FLEXOP Consortium, "The FLEXOP project," URL: https://flexop.eu/, 2015.

${ }^{5}$ Patartics, B., Luspay, T., Péni, T., Takarics, B., Vanek, B., and Kier, T., "Parameter varying flutter suppression control for the BAH jet transport wing," IFAC Proceedings Volumes (IFAC-PapersOnline), 2017.

${ }^{6}$ Kier, T. and Looye, G., "Unifying Manoeuvre and Gust Loads Analysis," International Forum on Aeroelasticity and Structural Dynamics, 2009.

${ }^{7}$ Stahl, P., Sendner, F.-M., Hermanutz, A., Rößler, C., and Hornung, M., "Mission and Aircraft Design of FLEXOP Unmanned Flying Demonstrator to Test Flutter Suppression within Visual Line of Sight," 17th AIAA Aviation Technology, Integration, and Operations Conference, 2017.

${ }^{8}$ Schulz, S., Aeroelastic Control Law Design Using Dierent Synthesis Methods, Master's thesis, University of Stuttgart, 2017.

${ }^{9}$ Ferede, E. and Abdalla, M., "Cross-sectional modelling of thin-walled composite beams," 55th AIAA/ASMe/ASCE/AHS/SC Structures, Structural Dynamics, and Materials Conference, 2014.

${ }^{10}$ Guyan, R. J., "Reduction of stiffness and mass matrices," AIAA Journal, 1964.

${ }^{11}$ Hauger, W., Schnell, W., and Gross, D., Technische Mechanik: Band 3: Kinetik, Vol. 217, Springer-Verlag, 2014.

${ }^{12}$ Reschke, C., Integrated flight loads modelling and analysis for flexible transport aircraft, Ph.D. thesis, University of Stuttgart, 2006.

${ }^{13}$ Hofstee, J., Kier, T., Cerulli, C., and Looye, G., "A Variable, Fully Flexible Dynamic Response Tool for Special Investigations (VARLOADS)," International Forum on Aeroelasticity and Structural Dynamics, 2003.

${ }^{14}$ Kier, T. and Hofstee, J., "Varloads-eine Simulationsumgebung zur Lastenberechnung eines voll flexiblen, freifliegenden Flugzeugs," Deutscher Luft-und Raumfahrtkongress, 2004.

${ }^{15}$ Kier, T., "Comparison of Unsteady Aerodynamic Modelling Methodologies with respect to Flight Loads Analysis," International Forum on Aeroelasticity and Structural Dynamics, 2001.

${ }^{16}$ Knoblach, A., Robust Performance Analysis for Gust Loads Computation, Ph.D. thesis, Hamburg University of Technology, 2015.

${ }^{17}$ Roger, K. L., "Airplane Math Modeling Methods for Active Control Design," AGARD-CP-228, 1977.

${ }^{18}$ Tewari, A., Aeroservoelasticity - Modeling and Control, Springer-Verlag, 2015. 